\title{
Produtividade e Eficiência Biológica de Sistemas de Produção de Gado de Corte de Ciclo Completo no Rio Grande de Sul ${ }^{1}$
}

\author{
Virgínia Beretta ${ }^{2}$, José Fernando Piva Lobato ${ }^{3}$, Carlos Guilherme Mielitz Netto ${ }^{4}$
}

\begin{abstract}
RESUMO - Um modelo estático determinístico, com base em resultados de pesquisas, avaliou por intermédio de simulações, a produtividade e a eficiência biológica de sistemas de ciclo completo de produção de bovinos de corte no Rio Grande do Sul, diferindo na idade das novilhas no primeiro parto (IP, meses) e dos novilhos ao abate (IA, meses): "Sistema Tradicional" (ST) IP48, IA54; "Sistema Melhorado" (SM), IP36, com duas idades de abate, IA36 (SM-I) e IA24 (SM-II); "Sistema Um Ano" (SU), IP24, com três idades de abate, IA36 (SU-I), IA24 (SU-II) e IA18 (SU-III). Para cada um deles foram avaliadas mudanças na taxa de natalidade (TN) entre 50 e $90 \%$. O ST foi baseado em campo nativo. Os outros sistemas introduziram o ajuste da carga sazonal do campo nativo e pastagens nativas melhoradas com espécies hibernais, para as categorias em evolução. A produção de peso vivo (PV) mostrou incrementos decrescentes com a TN, atingindo valores máximos de 73, 97, 129, 116, 149 e 151 kg/ha nos sistemas ST, SM-I, SM-II, SU-I, SU-II e SU-III, respectivamente. A TN ótima aumentou com a IP. A resposta a aumentos na TN entre 50\% e o ótimo mostrou uma tendência a crescer, quanto menor for a IA dos novilhos: 23,2\% (SU-III), 22,2\% (SM-II), 21,4\% (SU-II), 20,3\% (SM-I), 16,8\% (SU-I), e 15,1\% (ST). A eficiência biológica no SU-III com TN=80\% foi de 35,1 Mcal EM/kg PV. A melhoria simultânea dos índices reprodutivos e produtivos permitiu reduzir o custo energético por quilo de PV em 42,2\% em relação ao $\mathrm{ST}$ com $\mathrm{TN}=50 \%$.
\end{abstract}

Palavras-chave: eficiência biológica, gado de corte, produtividade, simulação, sistemas

\section{Productivity and Biological Efficiency of Alternative Beef Cattle Life-Cycle Production Systems in Rio Grande do Sul}

\begin{abstract}
A static and deterministic model, synthesizing published experimental information, was used to simulate the productivity and biological efficiency of beef cattle life-cycle production systems in Rio Grande do Sul, differing in heifers' age at first calving (IP, months) and steer age at slaughter (IA, months): "Traditional System" (ST), IP48, IA54; "Improved System" (SM), IP36, with two slaughter ages, IA36 (SM-I) and IA24 (SM-II); "One Year System" (SU), IP24, with three slaughter ages, IA36 (SU-I), IA24 (SU-II) and IA18 (SU-III). For each system, the variation on calving rate (TN) between 50 and $90 \%$ was evaluated. ST was based on native pasture. Other systems adjusted seasonal stocking rate in native pasture and used improved winter pastures with those animal categories pretended to be improved. Live weight production showed reduced increments with increasing TN, reaching maximal values of 73, 97, 129, 116, 149 and $151 \mathrm{~kg} /$ ha for systems ST, SM-I, SM-II, SU-I, SU-II and SU-III, respectively. Optimum TN increased with IP. Responses to increasing TN between $50 \%$ and optimal value, showed a tendency to increase with younger IA of steers: $23,2 \%$ (SUIII), 22,2\% (SM-II), 21,4\% (SU-II), 20,3\% (SM-I), 16,8\% (SU-I), and 15,1\% (ST). Biological efficiency for SU-III system with TN=80\% was of $35,1 \mathrm{Mcal} \mathrm{ME} / \mathrm{kg}$ of LW. Improving simultaneously, reproductive and productive coefficients, reduced LW production energetic cost in $42,2 \%$ with respect to cost in $\mathrm{ST}$ with $\mathrm{TN}=50 \%$.
\end{abstract}

Key Words: beef cattle, biological efficiency, productivity, simulation, systems

\section{Introdução}

A produção de gado de corte no Estado do Rio Grande do Sul é realizada em condições de pastejo, quase exclusivamente sobre pastagens nativas, sem considerar a capacidade de suporte das mesmas. Assim, a produtividade por unidade de área é baixa
(Maraschin \& Jacques, 1993; Quadros \& Lobato, 1996; Simeone \& Lobato, 1996).

Abundante pesquisa tem sido desenvolvida no estado dirigida à geração de novas técnicas que permitam melhorar esses indicadores de produtividade (Lobato, 1995; Moraes et al., 1995). Essa pesquisa, no entanto, tem tido um enfoque disciplinar, e na

\footnotetext{
${ }_{1}$ Parte da tese de Doutorado em Zootecnia apresentada à Universidade Federal do Rio Grande do Sul- UFRGS pelo primeiro autor. 2 Doutora, Departamento de Produção Animal e Pastagens, Estação Experimental "Mario A. Cassinoni", Faculdade de Agonomia, Universidade da República, C.P. 57 072, CEP: 60 000, Paysandú - Uruguai. E.mail: asimeone @ adinet.com.uy

${ }^{3} \mathrm{PhD}$, Professor Adjunto IV, Dep. de Zootecnia, Fac. de Agronomia - UFRGS. C.P. 776 - 90.001-970 - Porto Alegre, RS. E.mail: lobato@orion.ufrgs.br Bolsista do CNPq.

4 Doutor, Professor Adjunto III do Departamento de Economia Rural da UFRGS - Porto Alegre-RS. E.mail: mielitz@ vortex.ufrgs.br
} 
maioria das vezes, a avaliação da resposta à nova tecnologia tem-se restringido apenas à categoria objeto de estudo, sendo poucos os estudos considerando o impacto global das mesmas no sistema de produção (Cezar et al., 1996; Beretta, 1999; Pötter et al., 1998, 2000).

Os sistemas de produção pecuários são complexos, onde numerosos fatores (genótipo, pastagens, suplementos, sanidade, estrutura de preços de insumos e produtos, gerenciamento etc) interagem entre si, tornando difícil a predição da resposta global do sistema diante de inovações tecnológicas, quando se altera apenas um componente (Black et al., 1993). Dois trabalhos anteriores a este determinaram, por intermédio de simulações, que o impacto de melhorias dos índices reprodutivos (Beretta et al., 2002) e produtivos (Beretta et al., 2002) está na produtividade global e na eficiência biológica de sistemas especializados de cria, ou de recria e engorda, respectivamente, aasociado às respostas às mudanças em um indicador condicionadas por valores assumidos pelos demais coeficientes técnicos dos sistemas. É provável que respostas à incorporação das mesmas técnicas, porém em sistemas de produção de carne de ciclo completo, mudem em relação à registrada em sistemas especializados de cria ou de engorda.

O objetivo deste trabalho foi avaliar, por intermédio de um modelo quantitativo, representativo de um sistema de ciclo completo de pecuária de corte no Rio Grande do Sul, a produtividade física e a eficiência biológica de sistemas diferindo na intensidade de uso dos recursos e no valor atingido pelos indicadores reprodutivos e produtivos, como a taxa de natalidade, idade das novilhas ao primeiro parto e idade dos novilhos ao abate.

\section{Material e Métodos}

Foi avaliada mediante a simulação computacional a produtividade e a eficiência biológica de seis sistemas pecuários de ciclo completo caracterizados por uma melhoria gradativa nos indicadores reprodutivos e produtivos.

Os sistemas foram classificados em "Sistemas Tradicionais" (ST), "Sistemas Melhorados" (SM) e "Sistemas Um Ano" (SU), conforme a idade ao primeiro parto (IP) das novilhas aos 48,36 ou 24 meses, respectivamente. No ST foi considerado o abate dos novilhos aos 54 meses. Reduções na idade de abate (IA) para 36 e 24 meses foram consideradas no SM e SU e, também, uma redução para 18 meses no SU (Tabela 1). Para cada um destes sistemas foi avaliado o impacto de mudanças na taxa de natalidade $(\mathrm{TN})$ variando entre 50 e $90 \%$. As principais inovações tecnológicas consideradas, determinantes das mudanças nos coeficientes técnicos de produção de gado de corte, foram: i) o ajuste sazonal da carga animal em campo nativo; ii) o manejo da condição corporal (CC) das vacas de cria ao longo de ciclo reprodutivo; iii) a introdução de pastagens melhoradas de clima temperado e forrageiras de estação quente. A alimentação no ST foi exclusivamente em campo nativo. A redução da IP de 48 para 36 ou 24 meses foi viabilizada a partir da utilização de pastagens nativas melhoradas no período julho-novembro prévio ao primeiro acasalamento e durante o pré e pós-parto das vacas primíparas (Beretta \& Lobato, 1996; Rocha 1997; Pereira Netto \& Lobato, 1998).

As vacas de cria multíparas foram conduzidas sempre em campo nativo. As mudanças na TN foram quantificadas como função da CC das vacas ao parto e ao início do acasalamento (Orcasberro, 1991).

Para redução da IA foi considerada a utilização de pastagens nativas melhoradas durante o inverno/ primavera (Müller \& Primo, 1986; Quadros \& Maraschin, 1987; Albospino \& Lobato, 1994) e o ajuste da carga em campo nativo ao longo do ano conforme Moraes et al. (1995). Para o SU-III foi acrescentado o uso de pastagem de milheto (Pennisetum americanum) durante o verão pelos novilhos em terminação (Aita \& Restle, 1999).

\section{$O$ modelo}

Foi utilizado um modelo estático determinístico representando o ciclo anual de produção de carne, montado com base num sistema de equações lineares simultâneas resolvidas por planilha eletrônica (Beretta, 1999). A matriz geral de programação desse modelo simulou um sistema em equilíbrio para um período equivalente a um ano produtivo, incluindo todos os eventos reprodutivos do rebanho de cria, assim como as diferentes etapas de crescimento das categorias de recria para reposicão e engorda. A mesma foi estruturada em setores, cada um deles responsável pela quantificação dos diversos componentes do sistema de produção: estrutura do rebanho e transferência de animais entre categorias ao longo do ciclo produtivo, exigências energéticas animais, manejo alimentar e caracterização de recursos produtivos, uso da terra e carga. A partir desses dados, outro 
Tabela 1 - Caracterização dos sistemas de produção avaliados em termos da idade das novilhas ao primeiro parto e dos novilhos ao abate

Table 1 - Characterization of evaluated systems in terms of ages of heifers at first calving and steers at slaughter

\begin{tabular}{|c|c|c|c|c|}
\hline \multirow{2}{*}{$\begin{array}{l}\text { Idade das novilhas ao primeiro } \\
\text { parto (meses) } \\
\text { Heifers age at first calving (months) }\end{array}$} & \multicolumn{4}{|c|}{$\begin{array}{l}\text { Idade dos novilhos ao abate (meses) } \\
\text { Steers age at slaughter (months) }\end{array}$} \\
\hline & 54 & 36 & 24 & 18 \\
\hline 48 & $\begin{array}{c}\text { "Sistema } \\
\text { Tradicional" (ST) } \\
\text { Traditional } \\
\text { System }(S T)\end{array}$ & & & \\
\hline 36 & & $\begin{array}{c}\text { "Sistema } \\
\text { Melhorado I" } \\
\text { (SM-I) } \\
\text { Improved } \\
\text { system }(\text { SM-I) }\end{array}$ & $\begin{array}{c}\text { "Sistema } \\
\text { Melhorado II" } \\
\text { (SM-II) } \\
\text { Improved } \\
\text { system }(\text { SM-II) }\end{array}$ & \\
\hline 24 & & $\begin{array}{l}\text { "Sistema Um } \\
\text { Ano I" (SU-I) } \\
\text { One year } \\
\text { system }(S U-I)\end{array}$ & $\begin{array}{l}\text { "Sistema Um } \\
\text { Ano II" (SU-II) } \\
\text { One year } \\
\text { system }(S U-I I)\end{array}$ & $\begin{array}{l}\text { "Sistema Um } \\
\text { Ano III" } \\
\text { (SU-III) } \\
\text { One year } \\
\text { system (SU-III) }\end{array}$ \\
\hline
\end{tabular}

conjunto de equações fez a estimação da produtividade e eficiência biológica do sistema de produção correspondentes ao output do modelo. Os coeficientes da matriz de programação constituíram os inputs do modelo, que variaram conforme as técnicas utilizadas na produção de gado de corte. Estes coeficientes foram especificados para cada um dos seis sistemas avaliados, utilizando-se como principais fontes de informações resultados experimentais gerados no Estado do Rio Grande do Sul.

\section{Coeficientes técnicos}

Do sistema. O modelo foi dimensionado para uma área total de 1000 ha. A área da empresa é a única restrição do modelo. Foi considerado ser o potencial produtivo dos recursos naturais o mesmo nos diferentes sistemas, não sendo a produtividade limitada pela dotação natural de recursos, mas pela adoção de tecnologia. Foi considerado que os sistemas que utilizam pastagens de ciclo hiberno/primaveril as incorporam através da integração da pecuária com a lavoura (Moraes et al., 1995), implantadas sobre a resteva da lavoura de verão, com uma vida produtiva média de três anos.

Do rebanho. A estrutura do rebanho em cada sistema foi gerada a partir do fornecimento dos índices de natalidade e mortalidade, IP das novilhas de reposição e IA ou venda dos machos e fêmeas, taxa de descarte de vacas e touros, e taxa de utilização de touros. Além dos índices diferenciados para cada sistema (Tabela 1), em todos os casos foi considerado uma taxa de utilização de touros de $4 \%$ com $25 \%$ de reposição anual; uma taxa de mortalidade de bezerros de $4 \%$ no ST e SM e de $5 \%$ no SU, sendo $3 \%$ para animais de 6 a 24 meses e de $1 \%$ para idades maiores. Como estratégia de descarte, todas as vacas vazias em $1^{\circ}$ de abril saíram do rebanho de cria sempre que a disponibilidade de novilhas de reposição fosse maior ou igual à quantidade de vacas falhadas, fixando-se uma taxa de descarte mínima de $15 \%$.

A demanda energética para cada categoria animal do rebanho foi estimada no modelo com base nas equações do Afrc (1993), sendo função do genótipo, da TN, das taxas de ganhos sazonais para as diferentes categorias em crescimento (as que variam dependendo da IP e IA), dos pesos dos bezerros ao nascimento e ao desmame, e da produção de leite das vacas. A raça considerada foi Hereford, com peso vivo adulto das vacas de $420 \mathrm{~kg}$, equivalente a uma $\mathrm{CC}=3(1=$ magra; 5 = gorda para abate $)$. Para as vacas vazias foi considerado um peso médio de 339 $\mathrm{kg}$ no descarte. A CC nos distintos momentos fisiológicos variou conforme a TN do rebanho. A CC ao parto (CCP) e início de acasalamento (CCA) foi definida a partir das relações encontradas por Orcasberro (1991): $\mathrm{TN}<80 \%, \quad \%$ Prenhez $=$ - 
$86,7+41,6 x\left(R^{2}=0,996\right) ; \quad T N>80 \%$, \%Prenhez $=$ $60,66+4 x\left(R^{2}=0,65\right)$; sendo $x=C C A$. As variações em peso vivo assumiram uma equivalência de $50,8 \mathrm{~kg}$ por unidade de CC, valores esses reportados por Orcasberro (1991). A produção média diária de leite fixou-se em 3,16 kg/vaca adulta (Rovira, 1996), tendo as primíparas $80 \%$ deste valor. O peso médio dos bezerros ao nascimento foi de $30 \mathrm{~kg}$ e à desmama aos 180 dias de $150 \mathrm{~kg}$. Baseado nos dados reportados por Rovira (1996) e Nuñes Dominguez et al. (1991), o peso à desmama de bezerros filhos de vacas primíparas aos dois anos foi considerado $12 \%$ inferior ao dos bezerros filhos de vacas primíparas aos três ou quatro anos. Todos os sistemas mantiveram machos castrados, vendidos com um peso vivo médio ao abate de $380 \mathrm{~kg}, 440 \mathrm{~kg}$ e $450 \mathrm{~kg}$ aos 18, 24 ou mais de 24 meses, respectivamente. As vacas de descarte foram engordadas em pastagens melhoradas de junho a setembro, vendidas com $420 \mathrm{~kg}$, exceto as do $\mathrm{ST}$, as quais permaneceram até o outono seguinte para atingir os $420 \mathrm{~kg}$. Em todos os sistemas as bezerras em excesso foram vendidas ao desmame.

Do manejo alimentar. No modelo de simulação, o manejo alimentar foi definido mensalmente para cada uma das categorias do rebanho. A área necessária de cada recurso forrageiro (campo nativo, pastagem melhorada ou milheto), foi estimada a partir do balanço energético entre o aporte das forrageiras/hectare e a demanda das categorias que as utilizavam. $\mathrm{O}$ campo nativo foi caracterizado em termos de produção sazonal de forragem ( $\mathrm{kg}$ matéria seca/ ha), qualidade (digestibilidade) e taxa de utilização, segundo Corrêa (1993) e Apezteguia (1994). As pastagens melhoradas temperadas, misturas de Lolium multiflorum, Trifolium repens e Lotus corniculatus, foram caracterizadas segundo Quadros e Maraschin (1987), e o milheto conforme Silva et al., (1995). Foi considerado as pastagens hibernais permanecerem fechadas de meados de novembro até janeiro para favorecer a ressemeadura das espécies introduzidas.

\section{Saídas do modelo}

Produção de peso vivo. A produtividadade física do sistema é quantificada como os quilos de peso vivo produzidos por hectare de pastejo, a partir da equação:

Produçãode peso vivo $(\mathrm{kg} / \mathrm{ha})=$ vendas - compras + variaçãoem estoque Área de pastejo média anual

Considerados os sistemas como fechados e em equilibrio, não houve compras, e a variação dos quilos de peso vivo em estoque entre o início e o fim de cada ano produtivo foi zero, sendo, portanto, a produção anual de peso vivo igual às vendas do sistema nesse período. Adicionalmente, foi estimada a produtividade parcial do subsistema criador e invernador dentro do ciclo completo. Como produto da cria foi considerado o somatório em quilos de bezerros ao desmame, mais os das vacas de descarte por unidade de área destinada à cria (área de pastejo utilizada pelo rebanho de cria mais as novilhas para reposição). Na invernada, o produto foi quilos de peso vivo produzidos entre o desmame e o abate dos novilhos e durante a engorda das vacas de descarte, expressos em relação a área de pastejo utilizada por estas categorias.

Eficiência biológica. Este item quantificou a energia necessária por unidade de produto gerada no sistema. A mesma foi estimada a partir da relação entre a energia metabolizável (EM) consumida anualmente pelo rebanho e os quilos de peso vivo produzidos no sistema.

Taxa de desfrute (TD). Foi definida como a relação percentual entre o peso vivo vendido e o total mantido em estoque. Em sistemas em equilíbrio, este indicador é um bom estimador da duração do ciclo produtivo e da velocidade de circulação do capital investido no processo.

De forma complementar, o modelo forneceu informações descritivas para cada sistema (uso da terra, estoque e estrutura do rebanho, carga animal), as quais permitiram interpretar as diferenças entre sistemas em produtividade e eficiência.

\section{Resultados e Discussão}

\section{Caracterização das empresas}

Uso da terra. Na Tabela 2 é apresentado o uso da terra e a composição do rebanho quando os sistemas foram avaliados à mesma TN (80\%), a exceção do ST, o qual foi considerado como sistema de referência, com $50 \%$ de TN. Na medida em que os sistemas se intensificaram, houve uma redução da área total de pastejo destinada à atividade pecuária, e um aumento da área relativa de pastagens nativas melhoradas em relação ao campo nativo. A área de pastejo diminuiu associado ao aumento das áreas de pastagens melhoradas, já que mais áreas de lavouras foram necessárias para renovar as mesmas. Embora isto não afete diretamente à produtividade da pecuária (avaliada como produto por unidade de área), poderá condicionar o resultado econômico em nível de empresa, ao considerar como receita total a de- 
rivada da pecuária mais a do arrendamento da terra a terceiros para a realização de lavouras.

A redução da IP das novilhas para 36 meses e da IA dos machos para 36 meses, exigiu 13,3\% da área de pastejo formado por pastagens melhoradas (SM-I). A redução da IA em mais um ano, quase dobrou as exigências deste recurso forrageiro, atingindo $23,9 \%$ da área de pastejo (SM-II). Quando as pastagens melhoradas foram destinadas às novilhas para diminuir a IP para 24 meses ao invés de reduzir a IA, as mesmas passaram a ocupar $16,1 \%$ da área de pastejo (SU-I). Uma proporção máxima de $26,6 \%$ foi registrada em sistemas com IP e IA aos 24 meses (SU-II). A importância relativa das pastagens é maior na área destinada exclusivamente à invernada em relação à cria $^{5}$. Sistemas com IP aos 36 meses necessitaram de $4,9 \%$ da área criadora ocupado por pastagens melhoradas, enquanto aqueles com IP aos 24 meses, tiveram de aumentar a área de pastagens para 9,6\% da área de pastejo da cria. Já no que respeita à invernada,

Tabela 2 - Uso da terra, estoque e estrutura de rebanho em sistemas pecuários de ciclo completo diferindo na taxa de natalidade, idade ao primeiro parto e ao abate. (Área total de $1000 \mathrm{ha}$ )

Table 2 - Use of land, herd size and structure for life-cycle beef cattle systems differing in calving rate and age at first calving and slaughter. (Area 1000 ha)

\begin{tabular}{|c|c|c|c|c|c|c|c|}
\hline \multirow{3}{*}{$\begin{array}{l}\text { Coeficiente } \\
\text { Coefficient } \\
\text { Idade ao abate } \\
\text { Slaughter age }\end{array}$} & \multirow{3}{*}{$\begin{array}{c}\text { Unidade }^{2} \\
\text { Unit } \\
\text { Meses } \\
\text { Months }\end{array}$} & \multicolumn{6}{|c|}{$\begin{array}{l}\text { Sistemas } \\
\text { Systems }\end{array}$} \\
\hline & & \multicolumn{2}{|c|}{ SU } & \multicolumn{2}{|c|}{ SM } & \multicolumn{2}{|c|}{ ST } \\
\hline & & 18 & 24 & 36 & 24 & 36 & 54 \\
\hline $\begin{array}{l}\text { Uso da terra } \\
\text { Land use }\end{array}$ & & & & & & & \\
\hline $\begin{array}{l}\text { Área pastejo } \\
\text { Grazing area }\end{array}$ & $\% \mathrm{SS}$ & 93 & 92 & 95 & 93 & 96 & 100 \\
\hline $\begin{array}{l}\text { Área pastagens } \\
\text { Pastures area }\end{array}$ & $\% \mathrm{SP}$ & 21,6 & 26,6 & 16,1 & 23,9 & 13,3 & 0,0 \\
\hline $\begin{array}{l}\text { Área cria } \\
\text { Breeding area }\end{array}$ & $\% \mathrm{SP}$ & 83 & 71 & 57 & 75 & 61 & 59 \\
\hline $\begin{array}{l}\text { Área invernada } \\
\text { Steer area }\end{array}$ & $\% \mathrm{SP}$ & 17 & 29 & 43 & 25 & 39 & 41 \\
\hline $\begin{array}{l}\text { Estoque animal }{ }^{3} \\
\text { Herd size } \\
\text { Estrutura do rebanho } \\
\text { Herd roud estructure }\end{array}$ & $\begin{array}{l}\text { Animais } \\
\text { Animal }\end{array}$ & 1001 & 952 & 1022 & 950 & 927 & 918 \\
\hline $\begin{array}{l}\text { Touros } \\
\text { Bulls }\end{array}$ & $(\%)$ & 1,9 & 1,5 & 1,1 & 1,4 & 1,2 & 1,1 \\
\hline $\begin{array}{l}\text { Vacas cria } \\
\text { Breeding cows }\end{array}$ & $(\%)$ & 48,6 & 39,6 & 29,3 & 35,8 & 30,8 & 28,4 \\
\hline $\begin{array}{l}\text { Novilhas de reposição } \\
\text { Replacement heifers }\end{array}$ & $(\%)$ & 13,1 & 10,6 & 7,9 & 19,1 & 16,4 & 26,7 \\
\hline $\begin{array}{l}\text { Vaca de descarte } \\
\text { Cull cows }\end{array}$ & $(\%)$ & 12,8 & 10,4 & 7,7 & 9,4 & 8,1 & 8,9 \\
\hline $\begin{array}{l}\text { Invernada } \\
\text { Steers }\end{array}$ & $(\%)$ & 23,6 & 37,9 & 54,0 & 34,3 & 43,5 & 34,9 \\
\hline
\end{tabular}

${ }^{1}$ SU, SM, ST: sistema "Um Ano", "Melhorado" e "Tradicional", com primeiro parto das novilhas aos 24,36 e 48 meses, respectivamente. SU e SM tem $80 \%$ de natalidade e ST 50\%. ("One year", "Improved" and "Traditional" systems, with heifers'first calving at 24,36 or 48 months, respectively. SU and SM have $80 \%$ calving rate and ST 50\%).

2 SS: área total, SP: superficie de pastejo (SS: total area, SP: grazing area).

${ }^{3}$ Valor médio anual (Mean annual value).

${ }^{4}$ Estrutura do rebanho no mês de abril, após desmame dos bezerros (Herd structure in April, after calf weaning). ${ }^{5}$ Considera-se área criadora aquela ocupada pelo rebanho de cria mais as fêmeas de reposição, sendo a área invernadora a ocupada
pelos novilhos mais as vacas imbernadas.

R. Bras. Zootec., v.31, n.2, p.991-1001, 2002 (suplemento) 
as pastagens melhoradas representaram $56,5 \%$ da área de pastejo nos sistemas com IA aos 24 meses, diminuindo este valor para $17,7 \%$ em sistemas com IA aos 36 meses. Nos sistemas com IA aos 18 meses, a proporção de pastagens foi $45,7 \%$, mas foi acrescentado o uso de pastagem cultivada de verão, sendo mínimo o uso do campo nativo. Estes resultados mostraram as maiores exigências alimentares associadas à melhoria do resultado produtivo de engorda em relação à cria. A vantagem relativa de investir em uma ou outra opção é condicionada pelo impacto físico da nova tecnologia em cada segmento produtivo e pelo retorno econômico da mesma.

Estoque animal. Foi registrado uma tendência de aumento do estoque conforme a intensificação dos sistemas de produção. O estoque foi função do número de animais e da área média necessária para manter cada 100 bezerros nascidos, sujeitos a área da empresa (Tabela 2). Embora as duas primeiras variáveis diminuíssem ao melhorar os índices reprodutivos e produtivos, paralelamente foi registrado uma diminuição da área de pastejo. As simulações mostraram que enquanto no ST foi necessário manter 585 animais para cada 100 bezerros nascidos, no SU-III (abate aos 18 meses) foram necessários apenas 239 animais, uma redução de 59,1\%. A mesma tendência foi registrada para a área ocupada por cada um destes rebanhos: enquanto no ST foram necessárias 637 ha, no SU-III este valor caiu para 222 ha, valor equivalente a uma terça parte do ST. Esta queda na área é o resultado do menor número de animais em estoque para cada 100 bezerros nascidos e do aumento simultâneo da capacidade de suporte da base forrageira utilizada na alimentação animal em sistemas intensivos. Maior TN e menores IP e IA, assim como menores índices de mortalidade, reduziram o número de categorias improdutivas (vacas falhadas, novilhas de reposição, novilhos em mantença ou com baixas taxas de ganho) a serem mantidas no rebanho, reduzindo o estoque necessário para cada 100 bezerros produzidos anualmente (Mielitz Netto, 1979).

A intensificação gerou também mudanças na estrutura de categorias do rebanho (Tabela 2). Estas modificações estão de acordo com a evolução na composição do estoque descrita por Mielitz Netto (1979) para um rebanho de ciclo completo, quando são introduzidas, por intermédio da simulação, variações independentes na TN, IP e IA. Segundo este autor, o aumento na TN diminui o número de vacas necessário/100 bezerros nascidos, determinando aumento no número de animais das demais categorias, que passam a ocupar a área liberada pelas vacas falhadas. Porém, a introdução de mudanças conjuntas nos indicadores reprodutivos e produtivos, como acontece ao comparar o ST com o SM-I (abate aos 36 meses), confunde os efeitos provocados por mudanças independentes em cada um deles: embora a TN tenha aumentado de 50 para $80 \%$, a IP passado de 36 para 24 meses e IA de 54 para 36 meses, o rebanho de vacas de cria cresceu, passando a ocupar a área liberada pelas fêmeas de reposição de dois a três anos e pelo machos em crescimento de três a quatro e de quatro a quatro e meio anos. A expansão do rebanho de cria atingiu o valor máximo no sistema com IP aos 24 meses e IA aos 18 meses (48,6\% do estoque), onde a invernada representou apenas $23,1 \%$ do estoque.

As mudanças na composição do estoque se refletiram na proporção relativa da área de pastejo destinada à cria e à invernada, respectivamente, em cada sistema (Tabela 2). Este indicador é relevante, uma vez que,

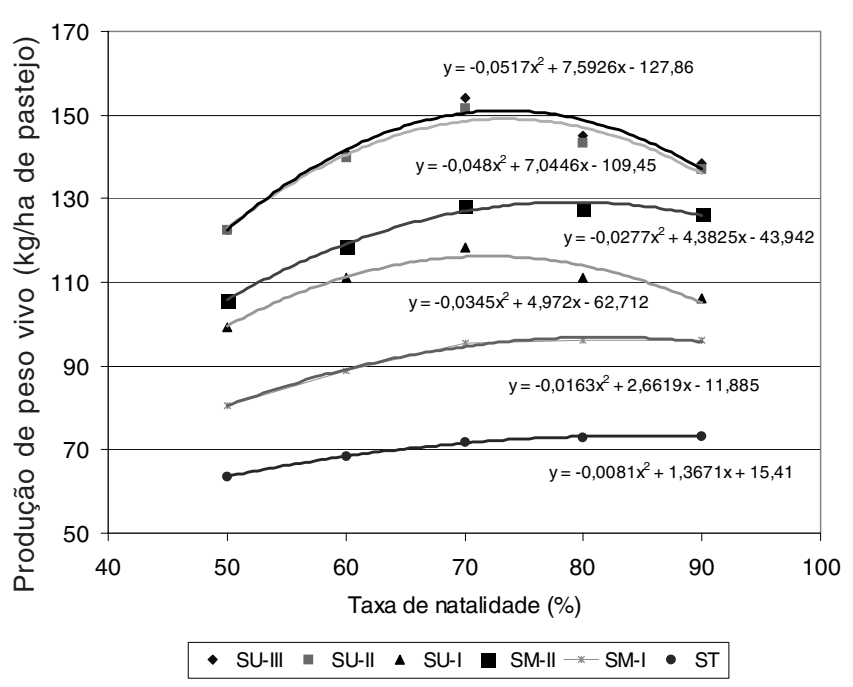

Figura 1 - Produção de peso vivo por hectare de pastejo em sistemas pecuários de ciclo completo diferindo na taxa de natalidade do rebanho de cria, na idade ao primeiro parto das fêmeas: quatro (ST), três (SM) ou dois anos (SU), e na IA dos novilhos: 54 (ST), 36 (SM-I e SU-I), 24 (SM-II e SU-II) e 18 meses (SU-III).

Figure 1 - Live weight gain per grazed hectare in life cycle production systems with different calving rate and heifer's first calving age: four (ST), three (SM) or two years old (SU) and steer age at slaughter: 54 (ST), 36 (SM-I e SU-I), 24 (SM-II e SU-II) and 18 months (SU-III). 
dado um potencial de produção diferenciado associado a cada uma das fases do ciclo de produção de bovinos de carne (Dickerson, 1978), a importância relativa que cada uma delas venha a ter no sistema de produção condicionará o resultado produtivo global da empresa.

\section{Produtividade}

Mudanças na TN variando entre 50 e $90 \%$ afetaram a produção de peso vivo, porém a magnitude e tipo de resposta variou conforme o valor assumido pelos demais coeficientes técnicos do sistema (Figura 1). Para todos os casos foi observada uma resposta de tipo quadrático, indicando incrementos decrescentes na produção de peso vivo/ha por unidade de aumento na TN. A maior produção potencial ${ }^{6}$ foi registrada peloSU-III (150,9 kg/ha), seguida pelo SU-II (149,0 kg/ha), SM-II (129,4 kg/ha), SU-I (116,4 kg/ ha), SM-I $(96,8 \mathrm{~kg} / \mathrm{ha})$ e ST $(73,1 \mathrm{~kg} / \mathrm{ha})$. A produtividade do ST com TN de $50 \%$ foi de $63,5 \mathrm{~kg} / \mathrm{ha}$, de forma que a otimização simultânea dos coeficientes reprodutivos e produtivos, sob as condições de manejo consideradas nas simulações, permitiu aumentar em $137,2 \%$ a produtividade dos sistemas pecuários de ciclo completo em relação aos indicadores atuais do Estado do Rio Grande do Sul.

A TN ótima ${ }^{7}(73,4 \%, 80,4 \%$ e 84,4\%) aumentou conforme foi retardada a IP das novilhas para 24, 36 e 48 meses, respectivamente). Esta tendência pode associar-se à importância relativa nos diferentes sistemas nas categorias de fêmeas de reposição em relação as vacas de descarte, tanto na área ocupada, quanto no número de animais em estoque, e à relação de substituição que se estabelece, como consequência disso entre o crescimento do rebanho de cria e o das categorias de invernada para cada novilha de reposição que sai do rebanho. Beretta et al. $(2001 ; 2002)$ reportaram a máxima produtividade para sistemas criatórios especializados ao atingirem valores de TN mais baixos em relação a sistemas de ciclo completo $(67,8 ; 68,3 ;$ e $72,8 \%$, com a IP aos 24 , 36 ou 48 meses, respectivamente). É provável que a engorda associada à cria valorize mais a produção de bezerros em relação a vaca de descarte no produto físico da empresa. O preço diferenciado que é pago pelas diferentes categorias pode, ainda, vir a modificar a TN ótima quando a mesma é avaliada em termos do resultado econômico da empresa.

$\mathrm{O}$ aumento da $\mathrm{TN}$ dentro de um mesmo sistema, desde $50 \%$ até atingir o valor ótimo, representou um crescimento da produtividade de $23,2 \%$ (SU-III), $22,2 \%$ (SM-II), 21,4\% (SU-II), 20,3\% (SM-I), 16,8\% (SU-I), e $15,1 \%$ (ST), registrando tendência de redução da resposta quanto maior for a IA dos novilhos. A relação de substituição de uma vaca de descarte por um novilho apareceu como mais vantajosa quanto mais eficiente foi o processo de crescimento e engorda. Isto mostra a interação entre o subsistema criador e o invernador dentro do ciclo completo, o que poderá vir a condicionar a ordem de introdução de melhorias dependendo da situação de início.

Quando avaliados os sistemas mais intensivos à uma mesma TN, o impacto produtivo da redução da IP de 36 para 24 meses diferiu segundo a IA dos machos. Em sistemas com $80 \%$ de TN e IA dos novilhos aos 36 meses (SM-I), reduzir em um ano a IP das novilhas (SU-I) aumentou a produção de peso vivo em $15,6 \%$. Este valor diminuiu para $11,7 \% \mathrm{em}$ sistemas com IA aos 24 meses e para 9,8\% em sistemas com IA aos 18 meses. A resposta à redução da IP de 36 para 24 meses porém, avaliada somente em nível da fase de cria, foi maior em relação ao ciclo completo: $17-18 \%$ de aumento nos quilos de bezerro desmamado mais as vacas de descarte por hectare.

Em síntese, foi observado que o impacto de uma mudança introduzida na fase de cria diminuiu quando avaliado em nível do ciclo completo de produção. A resposta produtiva $(\mathrm{kg} \mathrm{PV} / \mathrm{ha})$ à melhoria dos coeficentes reprodutivos foi condicionada pela IA dos novilhos, registrando-se que quanto menor foi esta variável, maior foi a resposta a aumentos da TN e menor o impacto resultante de antecipar a IP. Esta interação entre IP, TN e IA deve condicionar a adoção, independentemente de uma nova técnica, sem considerar as demais variáveis de manejo da empresa, destacando a importância da avaliação de cada inovação tecnológica no contexto do sistema produtivo a ser realizado, considerando o funcionamento global da empresa e não apenas as categorias diretamente afetadas. A prioridade na melhoria dos

\footnotetext{
6 Equivale à produção do sistema no ponto de máxima produtividade da função de resposta.

7 Taxa de natalidade que maximiza a produção de peso vivo por unidade da área, estimada a partir da derivada segundo a função de resposta de cada sistema.
}

\section{R. Bras. Zootec., v.31, n.2, p.991-1001, 2002 (suplemento)}


indicadores deverá ser avaliada à luz do resultado econômico. A produtividade dos sistemas melhorados superou em todos os casos à do $\mathrm{ST}$, independente da TN considerada. Quando a TN foi de 50\% no ST, a diminuição da IA de 54 para 36 meses e da IP de 48 para 36 meses (passar do ST para o SM-I), significou um aumento de $26,8 \%$ da produção de $\mathrm{kg} / \mathrm{PV} / \mathrm{ha}$ (63,5 vs 80,5), independentemente da TN. Quando a situação de partida foi o sistema com IP e IA aos 36 meses, a diminuição da IA para 24 meses (passar do SM-I para o SM-II) representou aumento da produção de $\mathrm{kg} \mathrm{PV} / \mathrm{ha}$ da ordem de $31 \%$, independentemente da TN do rebanho. Quando, em invés de investir na redução de IA, o sistema priorizou a melhoria dos índices reprodutivos, diminuindo a IP de 36 para 24 meses (passar do SM-I para o SU-I), a produtividade, avaliada na TN ótima, aumentou $24 \%$. A redução da IP de 36 para 24 meses em sistemas de ciclo completo só se justificou após ter diminuido a IA para 24 meses, sendo a resposta dependente da TN. Máximas respostas, da ordem de $18 \%$, foram registradas para $\mathrm{TN}=70 \%$ (passar do SM-II para o SU-II). Reduzir a IA para 18 meses em sistemas com IA e IP aos 24 meses (passar do SU-II para o SU-III), não apresentou maiores vantagens produtivas, apenas aumento de $1,3 \% \mathrm{em} \mathrm{kg/PV/ha.}$

A diminuição registrada na resposta produtiva na medida que avançou o processo de intensificação pode estar associada, nos sistemas de ciclo completo, à importância relativa que adquire a cria em relação à recria e engorda. As diferentes fases da pecuária de corte variam na sua produtividade potencial devido às caracterísiticas próprias dos processos fisiológicos de crescimento e reprodução envolvidos em cada caso (Dickerson, 1978). A produtividade da cria, considerados somente os quilos de bezerro desmamado/ha, foi sempre menor à da fase de engorda, acentuando-se as diferenças quanto mais eficiente foi o processo de crescimento e terminação (Tabela 3). Esta diferença na produtividade da cria em relação à engorda afetou a produção de peso vivo total, a qual é ponderada pela importância relativa das áreas de criação de invernada em cada sistema. Embora no SU-III registrou-se a maior produção de peso vivo na fase de engorda, o fato de o rebanho de cria ocupar $83 \%$ da área de pastejo (Tabela 2), limitou a produção de peso vivo total da empresa. No outro extremo, no $\mathrm{ST}$, a menor área relativa ocupada pelo rebanho de cria

Tabela 3 - Índices de produtividade, carga e eficiência biológica em sistemas pecuários de ciclo completo diferindo na taxa de natalidade, idade ao primeiro parto e ao abate (Área total de 1000 ha)

Table 3 - Liveweight production/ha, stocking rate and biological efficiency in life-cycle beef cattle systems differing in calving rate, age at first calving and slaughter. (Area 1000 ha)

\begin{tabular}{|c|c|c|c|c|c|c|c|}
\hline \multirow{3}{*}{$\begin{array}{l}\text { Coeficiente } \\
\text { Coefficient } \\
\text { Idade ao abate } \\
\text { Slaughter age }\end{array}$} & \multirow{3}{*}{$\begin{array}{c}\begin{array}{c}\text { Unidade }^{2} \\
\text { Unit }\end{array} \\
\text { Meses } \\
\text { Months }\end{array}$} & \multicolumn{6}{|c|}{$\begin{array}{l}\text { Sistemas } \\
\text { Systems }\end{array}$} \\
\hline & & \multicolumn{2}{|c|}{$\mathrm{SU}$} & \multicolumn{2}{|c|}{ SM } & \multicolumn{2}{|c|}{ ST } \\
\hline & & 18 & 24 & 36 & 24 & 36 & 54 \\
\hline $\begin{array}{l}\text { PV total } \\
\text { Total } L W\end{array}$ & $\mathrm{~kg} / \mathrm{ha} \mathrm{SP}$ & 145 & 143 & 111 & 128 & 96 & 64 \\
\hline $\begin{array}{l}\text { PVTD+VD } \\
W+C \text { Cull cow }\end{array}$ & $\mathrm{kg} / \mathrm{ha} \mathrm{SPC}$ & 77 & 81 & 81 & 70 & 70 & 38 \\
\hline $\begin{array}{l}\text { PV invernada } \\
\text { Steer } L W\end{array}$ & kg/ha SPI & 359 & 228 & 116 & 230 & 104 & 67 \\
\hline $\begin{array}{l}\text { Carga média anual global } \\
\text { Annual mean stocking rate }\end{array}$ & $\mathrm{UA} / \mathrm{ha}$ & 0,92 & 0,86 & 0,75 & 0,81 & 0,70 & 0,62 \\
\hline $\begin{array}{l}\text { Eficiencia biológica global } \\
\text { Global biological efficiency }\end{array}$ & Mcal EM/kg PV & 35,1 & 36,0 & 41,7 & 38,2 & 46,0 & 60,6 \\
\hline $\begin{array}{l}\text { CRA de } \mathrm{EM}(\mathrm{ST}=100) \\
\text { RAC of } M E(T S=100)\end{array}$ & $(\%)$ & 139 & 143 & 137 & 133 & 128 & 100 \\
\hline
\end{tabular}

1 SU, SM, ST: sistema "Um Ano", "Melhorado" e "Tradicional", com primeiro parto das novilhas aos 24, 36 e 48 meses, respectivamente. SU e SM tem 80\% de natalidade e ST 50\% ("One year", "Improved" and "Traditional" systems, with heifers' first calving at 24, 36 or 48 months, respectively. SU and SM have $80 \%$ calving rate and ST $50 \%$ ).

2 PV: peso vivo; TD: bezerro desmamado; VD: vaca de descarte; CRA: consumo relativo de energia metabolizável (EM) (IA: steer slaughter age; LW: live weight; W: weaned calf; RAC: relative annual consumption of metabolic energy [ME]).

3 SP: área de pastejo, SPC: área de pastejo da cria; SPI: área de pastejo da invernada; UA: unidade animal = $450 \mathrm{~kg}$ PV (SP: grazing area; SPC: Breeding, grazing area; SPI: growth and fattening grazing area; UA: animal unit $=450 \mathrm{~kg}$ live weight). 
(59\% da área de pastejo; Tabela 2), diluiu a baixa produtividade da fase de criação no produto total.

A maior produção de peso vivo/ha registrada na medida em que foram intensificadas os sistemas pecuários esteve associada a um aumento da carga por unidade de área de pastejo. O ST registou uma carga média anual de $0,62 \mathrm{UA} / \mathrm{ha}, 48,4 \%$ inferior ao máximo valor de $0,92 \mathrm{UA} /$ ha do SU-III. Os sistemas "Um Ano" combinaram altas UA/ha (SU-II: 0,86; SU-I: 0,75 UA/ha) e elevado desempenho individual, enquanto que, os melhorados comportaram valores intermediários (SM-II: 0,81; SM-I: 0,70). O aumento da carga sustentou-se na melhoria da capacidade de suporte da base forrageira, acontecendo uma mudança do que seria a ótima pressão de pastejo ao passar de um sistema para outro (Hodgson, 1990). O aumento da carga média em sistemas intensivos pode ser explicado, basicamente, pelo aumento da carga no manejo da invernada ao se reduzir a IA. Nos sistemas com abate aos 18 (SU-III) e 24 meses (SU-II e SM-II), a carga média anual para a invernada foi de 2,31 e $1,13 \mathrm{UA} / \mathrm{ha}$, respectivamente. No primeiro caso, uma alta carga responde ao manejo de uma única categoria de novilhos, exclusivamente sobre pastagens nativas melhoradas no inverno/primavera e pastagens cultivadas de verão. Nos sistemas com IA aos 24 meses, o aumento da carga passou pela utilização no inverno e primavera de pastagens melhoradas pelos bezerros desmamados, novilhos de sobre ano e vacas em engorda. No ST foi registrada uma carga para a invernada com IA aos 54 meses de $0,7 \mathrm{UA} / \mathrm{ha}$. A redução da IA para 36 meses (SU-I e SM-I), determinou a redução da carga da invernada para 0,65 UA/ha. Estes dois sistemas utilizaram pastagens melhoradas com os novilhos em terminação, mas as categorias restantes em crescimento foram manejadas em campo nativo. Dado que é necessário manter maiores taxas de ganho do que no ST, a carga em campo nativo diminuiu para atingir esse objetivo, diminuindo a carga média anual do sub-sistema invernador. O impacto da incorporação de pastagens sobre a carga média anual, diminuiu quando analisado em nível de todo o sistema, tendo como principais causas o peso relativo da área de engorda e os períodos em que as pastagens permaneceram fechadas para ressemeadura ou para a acumulação de forragem no início do outono.

\section{Eficiência biológica}

O custo energético por quilo de peso vivo produzido mostrou uma relação positiva com a IP e IA, e negativa com a TN (Tabela 3). Em sistemas de ciclo completo este coeficiente integra as ineficiências que acontecem na fase de cria e invernada. São escassos os trabalhos avaliando a eficiência do ciclo completo de produção, e, mais ainda, em condições de pastejo. Davis et al. (1994) reportam uma eficiência global do processo de produção de carne de 28,0 Mcal EM/kg de PV produzido, mais próximo dos valores registrados nos sistemas mais intensivos neste estudo. No SU-III, com $80 \%$ de TN, a EM consumida por quilo de peso vivo produzido foi $42,2 \%$ inferior da necessária no ST. A redução dos custos de mantença, associada à diminuição na proporção de categorias improdutivas do rebanho e à menor duração do ciclo produtivo ao se reduzir a IA, foi o principal componente a explicar a melhoria da eficiência biológica. A isto somou-se uma maior eficiência no uso da EM para os processos produtivos quando a qualidade da dieta melhorou (Afrc, 1993), basicamente devido a incorporação das pastagens melhoradas.

A demanda anual de energia em cada sistema aumentou conforme aumentou a TN e diminuiu a IP e IA, registrando-se no SU-II necessidades $43 \%$ superiores às do ST (Tabela 3). O maior peso vivo e $\mathrm{CC}$ das vacas ao aumentar a TN, assim como as maiores taxas de ganho das categorias em crescimento e engorda, elevou a demanda de EM por animal. Alta exigência individual, somado a maior número de cabeças em estoque (Tabela 2), determinaram maior consumo anual de energia quanto mais intensivo foi o sistema de produção. O maior consumo registrou-se para o SU-II. O SU-III demandou anualmente $4 \%$ menos EM do que o SU-II. O menor peso vivo médio dos novilhos ao abate ( $380 \mathrm{vs} 420 \mathrm{~kg}$ ) reduziu o custo total de mantença durante o período de engorda.

O aumento do consumo total de EM somado a melhoria da eficiência biológica de conversão da mesma, explicam o aumento da produtividade física quando foram melhorados os índices reprodutivos e produtivos dos sistemas pecuários. No entanto, esta maior demanda energética afeta diretamente os custos de alimentação (Beretta, 1999), o que poderá vir a condicionar o benefício econômico das diferentes alternativas tecnológicas.

\section{Taxa de desfrute}

A TD, quantificando a proporção de cabeças que saem do sistema em relação ao total do estoque mantido no mesmo, aumentou na medida que o processo produtivo foi intensificado, passando de $15,8 \%$

R. Bras. Zootec., v.31, n.2, p.991-1001, 2002 (suplemento) 
no ST para 46,8\%, 37,6\%, 27,6\% 34,3\% e $29,1 \%$ no SU-III, SU-II, SU-I, SM-II, e SM-I, repectivamente. A TD para a pecuária brasileira, no período 1988 1998 variou entre $17,1 \%$ e $21,8 \%$ (Nehmi Filho, 1998). Pötter et al. (1998) obtiveram a mesma tendência, reportando valores de $13,6 \%, 28,6 \%$ e $34,7 \%$, para o ST, SM e SU, respectivamente, os dois últimos com abate aos 24 meses.

Os valores assumidos pelos coeficientes técnicos afetaram diretamente a TD. O baixo desfrute do ST foi explicado pela baixa TN (50\%) e elevada IA (54 meses). Ambos fatores determinaram um baixo número de vacas de descarte e de animais prontos para abate em relação ao estoque. Comparando sistemas extremos, do total de animais em processo de crescimento e engorde, no ST apenas $25 \%$ saíram anualmente do sistema, enquanto que no SU-III $100 \%$ foi vendido no mesmo ano. Na medida que são sistemas em equilíbrio, e portanto, o tamanho do rebanho se mantêm estável, uma elevada TD denotou uma baixa duração do ciclo produtivo, originando maior velocidade de circulação do capital investido.

A composição das vendas por categoria variou entre sistemas. As vacas de invernada que no ST representaram $48 \%$ dos quilos de carne vendidos caíram para 27 a $35 \%$ nos sistemas mais intensivos de produção. Em sistemas com alta TN, houve substituição de vacas de descarte por bezerras de desmame, uma vez que a produção das últimas superou as necessidades de reposição. Isto terá efeito direto na receita bruta da empresa, uma vez que o preço por quilo de peso vivo vendido varia para as diferentes categorias (Nehmi Filho, 1998). Estes resultados destacam a importância relativa que pode vir a ter a estratégia de descarte, colocando a questão de qual taxa de prenhez que maximiza o resultado econômico, não sendo, necessariamente, a mesma que maximiza o resultado produtivo.

\section{Conclusões}

A melhoria dos índices reprodutivos (taxa de natalidade e idade das novilhas ao primeiro parto) e produtivos (idade de abate dos novilhos) aumentou a produtividade física e melhorou a eficiência biológica de sistemas de ciclo completo de produção de carne bovina no Rio Grande do Sul.

A resposta dos sistemas à melhoria de apenas um indicador, foi condicionada pelo valor assumido pelos demais coeficientes técnicos do sistema, de maneira que o impacto produtivo associado a uma inovação tecnológica (introdução de pastagens melhoradas), deverá necessáriamente ser avaliado no contexto produtivo de cada empresa pecuária.

Na procura do aumento da produtividade de sistemas "Tradicionais" de ciclo completo de produção de gado de corte, com $50 \%$ de natalidade, primeiro parto das novilhas aos 48 meses e abate dos novilhos aos 56 meses, a maior resposta biológica foi registrada conforme avançou a intensificação: i) redução da idade ao primeiro parto de 48 para 36 meses; ii) redução da idade de abate de 56 para 36 meses; iii) aumento da taxa de natalidade de 50 para $75 \%$. Sistemas com engordes mais intensivos (abate aos 18 meses), não apresentaram maiores vantagens, do ponto de vista biológico, quando inseridos em sistemas fechados de ciclo completo.

\section{Literatura Citada}

AGRICULTURAL RESEARCH COUNCIL - AFRC. Energy and protein requirements of ruminants. An advisory manual prepare by the AFRC technical Committee on Responses to Nutrients. Wallingford: CAB International, 1993. 159p.

AITA, V.; RESTLE, J. Produção animal em pastagens de estação quente. In: REUNIÃO ANUAL DA SOCIEDADE BRASILEIRA DE ZOOTECNIA, 36., 1999, Porto Alegre. Anais... Porto Alegre: Sociedade Brasileira de Zootecnia, 1999. p.13.

ALBOSPINO, B.H.; LOBATO, J.F.P. Efeitos do desmame precoce de bezerros no desempenho até os $24-26$ meses de idade. Revista Brasileira de Zootecnia, v.23, n.4, p.565575, 1994.

APEZTEGUíA, E. Potencial produtivo de uma pastagem natural do Rio Grande do Sul, submetida a distintas ofertas de forragem. Porto Alegre: Universidade Federal do Rio Grande do Sul, 1994. 169p. Dissertação (Mestrado em Agronomia) - Universidade Federal do Rio Grande do Sul, 1994.

BERETTA, V. Avaliação bioeconômica de sistemas alternativos de produção de gado de corte no Rio Grande do Sul. Porto Alegre: Universidade Federal do Rio Grande do Sul, 1999. 204 p. Tese (Doutorado em Zootecnia) - Universidade Federal do Rio Grande do Sul, 1999.

BERETTA, V.; LOBATO, J.F.P. Efeitos da ordem de utilização de pastagens melhoradas no ganho de peso e comportamento reprodutivo de novilhas de corte. Revista Brasileira de Zootecnia, v.25, n.6, p.1205-1215, 1996.

BERETTA, V.; LOBATO, J.F.P.; MIELITZ NETTO, C.G. Produtividade e eficiência biológica de sistemas pecuários criadores diferindo na idade das novilhas ao primeiro parto e na taxa de natalidade do rebanho de cria no Rio Grande de Sul. Revista Brasileira de Zootecnia, v.30, n.4, p.1278-1288, 2001.

BERETTA, V.; LOBATO, J.F.P.; MIELITZ NETO, C.G. Produtividade e eficiência biológica de sistemas de recria e engorda de gado de corte no Rio Grande do Sul. Revista Brasileira de Zootecnia, v.31, n.2, p.696-706, 2002.

BLACK, J.L.; DAVIES, G.T.; FEMING, F.F. Rol of computer simulation in the applications of knowledge to animal 
industries. Australian Journal of Agriculture Research, v.44, n.3, p.541-555, 1993.

CEZAR, I.M.; EUCLIDES FILHO, K.; MARTINS, C.I. Novilho precoce: reflexos na eficiência e conomicidade do sistema de produção. Campo Grande: Empresa Brasileira de Pesquisa e Agropecuária/Centro Nacional de Pesquisa de Gado de Corte, 1996. 31p. (Documentos, 66).

CORRÊA, F.L. Produção e qualidade de uma pastagem nativa do Rio Grande do Sul sob níveis de oferta de forragem a novilhos. Porto Alegre: Universidade Federal do Rio Grande do Sul, 1993. 165p. Dissertação (Mestrado em Agronomia) - Universidade Federal do Rio Grande do Sul, 1993.

DAVIS, K.C.; TESS, M.W.; KRESS, D.D et al. Life cycle evaluation of five biological types of beef cattle in a cowcalf range production system: II. Biological and economic performance. Journal of Animal Science, v.72, n.10, p.2591-2598, 1994.

DICKERSON, G.E. Animal size and efficiency: basic concepts. Animal Production, v.27, n.3, p.367, 1978.

HODGSON, J. Grazing management: science into practice. New York: Longman Scientific \& Technical, 1990. 203p.

LOBATO, J.F.P. Produção e manejo de bovinos de corte. In: REUNIÃO ANUAL DA SOCIEDADE BRASILEIRA DE ZOOTECNIA, 32., 1995, Brasília. Anais... Brasília: Sociedade Brasileira de Zootecnia, 1995. p.405-414.

MARASCHIN, G.E.; JACQUES, A.V.A. Grassland opportunities in the subtropical region of South America. In: INTERNATIONAL GRASSLAND CONGRESS, 17. 1993, Palmerston North. Proceedings... Palmerston North: New Zealand Grassland Association, 1993. p.1977-1981.

MIELITZ NETTO, C.G.A. Análise das mudanças de alguns coeficientes técnicos na criação de bovinos de corte no Rio Grande do Sul. Porto Alegre: Universidade Federal do Rio Grande do Sul, 62p. Dissertação (Mestrado em Economia Rural) - Universidade Federal do Rio Grande do Sul, 1979.

MORAES, A.; MARASCHIN, G.E.; NABINGER, C. Pastagens nos ecossistemas de clima subtropical: pesquisas para o desenvolvimento sustentável. In: SIMPÓSIO SOBRE PASTAGENS NOS ECOSSISTEMAS BRASILEIROS. Anais... Brasília: Sociedade Brasileira de Zootecnia, 1995. p.147-200.

MÜLLER, L.; PRIMO, A.T. Influência do regime alimentar no crescimento e terminação de bovinos e na qualidade da carcaça. Pesquisa Agropecuária Brasileira, v.21, n.4, p.445-452, 1986.

NEHMI FILHO, V.A. El mercado de carnes en el Mercosur. Situación y perspectivas. Revista de Fucrea, n.194, p.7-10, 1998.

NUÑEZ-DOMINGUEZ, R.; CUNDIFF, L.V.; DICKERSON, G.E. et al. Lifetime production of beef heifers calving first at two vs. three years of age. Journal of Animal Science, v.69, n.9, p.3467-3479, 1991.

ORCASBERRO, R. Estado corporal, control del amamentamiento y performance reproductiva de rodeos de cria. In: PASTURAS Y PRODUCCIÓN ANIMAL EN AREAS DE GANADERÍA EXTENSIVA. Montevideo: INIA, 1991. p.158-163 (Série Técnica, 13)
PEREIRA NETO, O.A.; LOBATO, J.F.P. Efeitos da ordem de utilização de pastagens nativas melhoradas no desenvolvimento e comportamento reprodutivo de novilhas de corte. Revista Brasileira de Zootecnia, v.27, n.1, p.60-65, 1998.

PÖTTER, L.; LOBATO, J.F.P.; MIELITZ NETTO, C.G.A. Produtividade de um modelo de produção para novilhas de corte primíparas aos dois, três e quatro anos de idade. Revista Brasileira de Zootecnia, v.27, n.3, p.613619, 1998.

PÖTTER, L.; LOBATO, J.F.P.; MIELITZ NETTO, C.G.A. Análises econômicas de modelos de produção com novilhas de corte primíparas aos dois, três e quatro anos de idade. Revista Brasileira de Zootecnia, v.29, n.3, p.861-870, 2000.

QUADROS, S.L.F.; LOBATO, J.F.P. Efeito da lotação no comportamento reprodutivo de vacas de corte primíparas. Revista Brasileira de Zootecnia, v.25, n.1, p.22-35, 1996.

QUADROS, F.L.F.; MARASCHIN, G.E. Desempenho animal em misturas de espécies forrageiras de estaçao fria. Pesquisa Agropecuária Brasileira, v.22, n.5, p.535-541, 1987.

ROCHA, M.G. Desenvolvimento e características de produção e reprodução de novilhas primíparas aos dois anos de idade. Porto Alegre: Universidade Federal do Rio Grande do Sul, 1997. 247p. Tese (Doutorado em Agronomia) - Universidade Federal do Rio Grande do Sul, 1997.

ROVIRA, J. Manejo reproductivo de los rodeos de cría en pastoreo. Montevideo: Hemisferio Sur, 1996. 288p.

SILVA, A.W.; MACEDO, A.F.; FRANCISCATO, C. Produção e matéria seca de milheto, sorgo sudão e Teossinto, sob diferentes épocas de semeadura no Planalto Serrano Catarinense. In: REUNIÃO ANUAL DA SOCIEDADE BRASILEIRA DEZOOTECNIA, 32., 1995, Brasília. Anais... Brasília: Sociedade Brasileira de Zootecnia, 1999. p.13.

SIMEONE, A.; LOBATO, J.F.P. Efeito da lotação animal em campo nativo e do controle da amamentação no comportamento reprodutivo de vacas de corte primíparas. Revista Brasileira de Zootecnia, v.25, n.6, p.1216-1227, 1996.
Recebido em: 23/07/01 Aceito em: 12/01/02 